\title{
O que os dados têm a dizer sobre a relação desigualdade de renda e crescimento econômico para o Nordeste do Brasil? Evidências a partir de modelos semiparamétricos
}

\author{
Paulo de Andrade Jacinto* \\ César Augusto Oviedo Tejada** \\ Erik Alencar de Figueiredo**
}

\begin{abstract}
RESUMO - A relação entre a distribuição de renda e o crescimento econômico ocupa um espaço significativo na análise econômica. Kuznets (1955) postulou a existência de uma relação na forma de um $U$ invertido, indicando que, inicialmente, a desigualdade no curto prazo aumenta com o crescimento econômico e, no longo prazo, decresce a partir de um "turning point". Esse padrão ficou conhecido como a curva de Kuznets. Entretanto, esse padrão nem sempre foi constatado nos inúmeros estudos realizados. Com intuito de contribuir com essa literatura, o presente trabalho investiga a validade da hipótese de Kuznets para os municípios da região nordeste do Brasil nos anos de 1991 e 2000. A relação é testada para diversas formas funcionais e para duas medidas de desigualdade, a metodologia utilizada compreende os modelos paramétricos e, de modo inovador no Brasil para testar a curva de Kuznets, o uso de modelos semiparamétricos. A vantagem dos modelos semiparamétricos reside no fato de não ser necessário impor nenhuma estrutura sobre o comportamento dos dados. Além disso, ele permite, de maneira indireta, validar ou não os resultados obtidos pelos modelos paramétricos. Os resultados sugerem que a hipótese de U invertido não é refutada tanto pelos modelos paramétricos quanto pelo semiparamétricos.
\end{abstract}

Palavras-chaves: Kuznets. Semiparamétrico. Desigualdade. Crescimento econômico. Renda.

\section{INTRODUÇÃO}

A relação entre a distribuição de renda e o processo de crescimento econômico ocupa um espaço significativo no pensamento econômico. $O$ vínculo entre esses dois fenômenos foi relatado de maneira clara por Simon Kuznets, em 1955. O seu insight partiu de duas questões importantes acerca do crescimento econômico: (i) A desigualdade na distribuição de renda aumenta ou diminui à medida que ocorre o crescimento econômico? (ii) Quais são os fatores que determinam a desigualdade de renda no longo prazo?

\footnotetext{
* Doutor em Economia pela Universidade Federal do Rio Grande do Sul, professor do Programa de Pósgraduação em Economia da Pontifícia Universidade Católica do Rio Grande do Sul. Endereço eletrônico: paulo.jacinto@pucrs.br.

* Doutor em Economia pela Universidade Federal do Rio Grande do Sul, professor adjunto da Universidade Federal de Pelotas. Endereço eletrônico: cesartejada9@hotmail.com.

*** Doutor em Economia pela Universidade Federal do Rio Grande do Sul, professor adjunto I da Universidade Federal da Paraíba. Endereço eletrônico: eafigueiredo@msn.com.
} 
Essas questões evidenciaram a sua preocupação com o grau de desigualdade na distribuição de renda, cuja origem poderia estar associada ao crescimento econômico. Com base na evidência de dados de séries de tempo, Kuznets (1955) postulou a existência de uma relação na forma de um U invertido, indicando que, inicialmente, o padrão de desigualdade no curto prazo aumenta com o crescimento econômico e, no longo prazo, decresce a partir de um "turning point". Esse padrão ficou conhecido na literatura econômica como a curva de Kuznets.

Ao considerar que a proposição de Kuznets possa ser estendida para compreender o desenvolvimento em regiões ou municípios e que a região Nordeste do Brasil tem apresentado altas taxas de crescimento médio do produto interno bruto per capita para os nove estados no período de 1970 a $1989^{1}$, empiricamente, parece que essas informações são indícios para validar a existência do processo descrito por Kuznets para essa região. Caso contrário, que inferências podem ser feitas a partir do crescimento econômico desse período?

Assim, o presente trabalho tem por objetivo verificar a hipótese do U invertido entre desigualdade e crescimento econômico para os municípios da região Nordeste do Brasil no período 1991-2000. Será utilizada a abordagem semiparamétrica, cuja principal vantagem reside no fato dela permitir que os dados por si mesmo revelem a verdadeira relação entre as variáveis sem impor nenhuma estrutura. Além dessa introdução, o trabalho foi organizado em mais três seções. Na próxima, será apresentado, de forma sucinta, o processo de Kuznets e suas propriedades. Na seção três são descritos os dados, a metodologia e os resultados para a curva de Kuznets. A última seção é reservada às conclusões do trabalho.

\section{CURVA DE KUZNETS: PROCESSO DE KUZNETS E SUAS PROPRIEDADES}

A hipótese de uma curva na forma de um $U$ invertido na relação entre a desigualdade e crescimento econômico é devida aos trabalhos de caráter empíricos de Simon Kuznets. É o resultado do seu interesse em mensurar e analisar os dados relacionados ao crescimento econômico. Ele parte da suposição de uma economia com apenas dois setores de atividades: setor urbano e setor agrícola; de que a renda per capita média da população rural é menor do que a da população urbana; de que o percentual da renda do setor rural sobre a

\footnotetext{
${ }^{1}$ Utilizando dados de nove estados nordestinos no período 1970-1998, Barreto, Jorge Neto e Tebaldi (2201), mostraram que há uma relação direta na qual os estados com maior PIB nas décadas de 70, 80 e 90 são aqueles que tendem a possuir grau elevado de concentração.
}

66 
renda total diminui ao longo dos anos e, de que a desigualdade de renda na população rural é inferior a observada na população urbana.

Em seu trabalho seminal de 1955, ele mostrou como as mudanças intersetoriais de mão de obra da agricultura para setores não agrícolas, nesse caso, urbanos, com um maior padrão de renda per capita produziriam um padrão em que a desigualdade de renda aumentaria, inicialmente, para depois reduzir. Alguns anos mais tarde, em 1963, ele apresentou evidências empíricas adicionais que levaria a postular uma curva que iria levar o seu nome. Ela seria o resultado de uma migração da área rural para a urbana gerando uma relação entre a desigualdade e desenvolvimento econômico na forma de U invertido. Por essa curva, um padrão de desigualdade primeiro aumentaria e a partir de um determinado ponto diminuiria em relação ao crescimento econômico.

Em termos gerais, em seu modelo, Kuznets (1955) partiu da ideia de uma economia dual, em que $x$ representa a proporção da população dedicada ao setor moderno, indicando assim o nível de desenvolvimento, enquanto $\mu_{i}$ e $I_{i}(i=1,2)$ são as rendas médias e os níveis de desigualdade setoriais e assume que:

$$
\theta=\frac{\mu_{1}}{\mu_{2}}>1 \quad \text { e } \quad \frac{I_{1}}{I_{2}}>1
$$

A hipótese segundo a qual as forças que conduzem a desigualdade predominam durante as primeiras fases do crescimento pode ser formulada como:

$$
\left[\frac{\partial I}{\partial x}\right]_{x=0}>0
$$

Enquanto a existência de um turning point equivale à condição de:

$$
\left[\frac{\partial I}{\partial x}\right]_{x=1}<0
$$

A primeira condição informa que a desigualdade aumenta nas primeiras fases do crescimento, enquanto a segunda reflete a existência de um ponto de reversão, passando, portanto, a desigualdade a ser em última instância uma função decrescente. Não se espera que o U invertido seja "simétrico", dado que nas fases mais elevada de crescimento e ao final da migração, a desigualdade não seria igual à observada na fase inicial do processo. 


\section{DESIGUALDADE E CRESCIMENTO ECONÔMICO: O QUE OS DADOS TÊM}

A DIZER?

A maior parte da teoria econômica não identifica uma forma funcional específica para a relação entre a variável dependente e a variável explicativa numa regressão. Com intuito de evitar má especificação da estrutura paramétrica, nessa seção serão apresentados os resultados da estimação de uma curva de Kuznets para os municípios da região Nordeste do Brasil com o uso de um modelo semiparamétrico.

\subsection{BASE DE DADOS}

A forma mais adequada para verificar a existência da curva de Kuznets para os municípios da região Nordeste do Brasil deveria ser por meio de um estudo individual de cada município ao longo do tempo, porém em virtude da inexistência de informações que permitam construir séries longas de índices de desigualdade e renda per capita, optou-se pela comparação em diferentes estágios do desenvolvimento para estudar a sua evolução por meio de um painel de dados. Trata-se do mesmo procedimento observado nos estudos empíricos mais recentes que buscam verificar a validade ou não da hipótese da curva na forma de U invertido.

As informações utilizadas para estimar a curva de Kuznets foram obtidas do Atlas de Desenvolvimento Humano (2003) - PNUD, elaborado pela Fundação João Pinheiro e referem-se aos municípios da região nordeste do Brasil relativo aos anos 1991 e 2000. Na construção da base a ser utilizada foram considerados os ajustes para as emancipações municipais que porventura ocorreram ao longo desse período e os valores monetários da renda per capita encontram-se expressos em termos reais de 1 de agosto de 2000.

\subsection{EVIDÊNCIAS DA CURVA DE KUZNETS A PARTIR DE MODELOS SEMIPARAMÉTRICOS}

Nesta subseção serão explorados os resultados de um modelo semiparamétrico. A vantagem desse método reside no fato de que ele "permite os dados falarem por si mesmo". Trata-se de uma análise mais flexível, na qual as técnicas de regressão semiparamétrica também podem ser usadas com a finalidade de verificar a validade ou não de um modelo paramétrico. Ou seja, o procedimento de regressão não paramétrica poderia ser considerado 
como se fosse um estágio final de uma análise de dados ou um degrau no caminho confirmatório do processo de Kuznets.

A especificação não-paramétrica univariada mais simples para a relação entre índices de desigualdade de renda e renda pode ser expressa por

$$
y_{i}=f\left(x_{i}\right)+\varepsilon_{i} .
$$

onde $y_{i}$ representa os índices de desigualdade, $x_{i}$ a renda per capita e suas transformações ou proporção da população urbana e $\varepsilon_{i}$ um erro com média zero e variância constante. Ao contrário dos modelos paramétricos, a função $f\left(x_{i}\right)$ que descreve a relação entre a desigualdade de renda e o desenvolvimento econômico não é especificada ex ante. A $f\left(x_{i}\right)=E(y \mid x)$ constitui uma função de alisamento e as inferências para essa função $f$ são amplamente discutidas na literatura ${ }^{2}$. No presente trabalho, ela será estimada a partir da generalização dos Splines proposta por French et alli (2001). Especificamente, o modelo para $f\left(x_{i}\right)$ é dada pela equação

$$
\begin{aligned}
& f(x)=\beta_{0}+\beta_{1} x+\ldots \ldots \ldots . .+\beta_{m-1} x^{m-1}+\sum_{k=a}^{K} u_{k}\left|x-K_{k}\right|^{2 m-1} \\
& \quad \text { Para } m=1,2,3, \ldots \ldots . \text { e com } \\
& u=\left(u_{1}, u_{2}, \ldots \ldots \ldots ., u_{k}\right)^{\prime} \sim N\left(0, \sigma_{u}^{2} \Omega^{-1 / 2}\left(\Omega^{-1 / 2}\right)^{\prime}\right), \quad \Omega=\left[\left|K_{k}-K_{k^{\prime}}\right| 1 \leq k, k^{\prime} \leq K \mid\right]
\end{aligned}
$$

$\mathrm{Na}$ estratégia de estimação será considerada uma relação univariada entre os índices de desigualdade e a renda, expressada pela regressão não-paramétrica dada pela equação (1).

Os resultados podem ser sintetizados no Gráfico 1. Ela apresenta o alisamento Spline para a relação entre desigualdade e renda per capita (painéis “a" e "b”), e desigualdade proporção da população urbana (painéis "c" e "d"). Como pode ser visto, a curva de Kuznets no formato de U-invertido se verifica para relação índice de desigualdade L-theil bem como coeficiente Gini (gráfico “a” e "b”). O que os dados têm a dizer sobre a relação entre desigualdade de renda e crescimento econômico? Em resumo, ao fazer uso dos modelos semiparamétricos os resultados corroboram com a existência de uma curva no formato de $\mathrm{U}$ invertido. Apesar do baixo crescimento econômico observado nessa região para o período em estudo, esses resultados confirmam a trajetória de queda na desigualdade de renda que vem ocorrendo nessa região ao longo dos últimos anos mesmo que ainda seja lenta ao ser comparada com outras regiões do país.

\footnotetext{
2 Para detalhes, ver Ruppert e Carroll (2000).
} 
O trabalho de Ahluwalia (1976) é uma importante referência entre os estudos que buscaram validar a hipótese da curva de Kuznets. Um prova disso está na especificação sugerida por ele, o polinômio de $2^{\circ}$. grau, que tem sido o ponto de partida em inúmeros estudos que buscam evidências para a hipótese de Kuznets e que foi empregado nas subseções acima. Contudo, grande parte dos estudos não leva em conta que as evidências favoráveis a existência de uma curva na forma de U-invertido, no estudo de Ahluwalia (1976), se devem, não apenas ao fato de estimar uma fórmula reduzida, mas ao fato de fazer isso para a população de cada país da sua amostra dividida em cinco quintis.

(a)

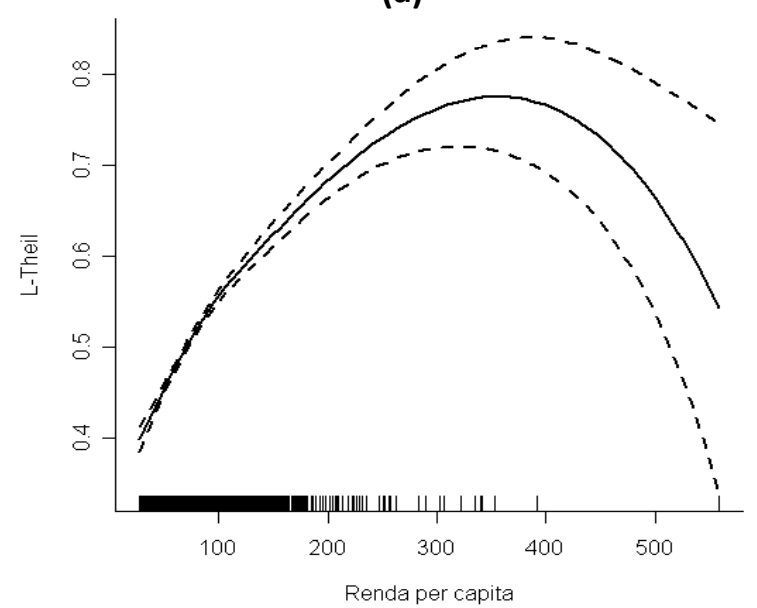

(c)

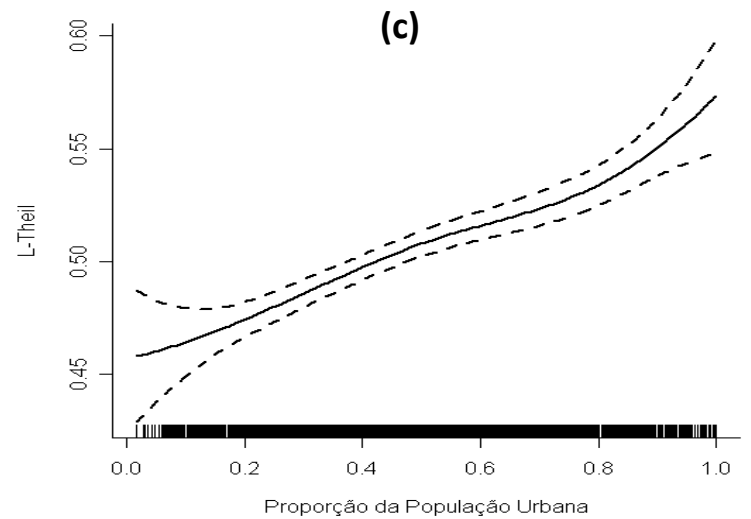

(b)

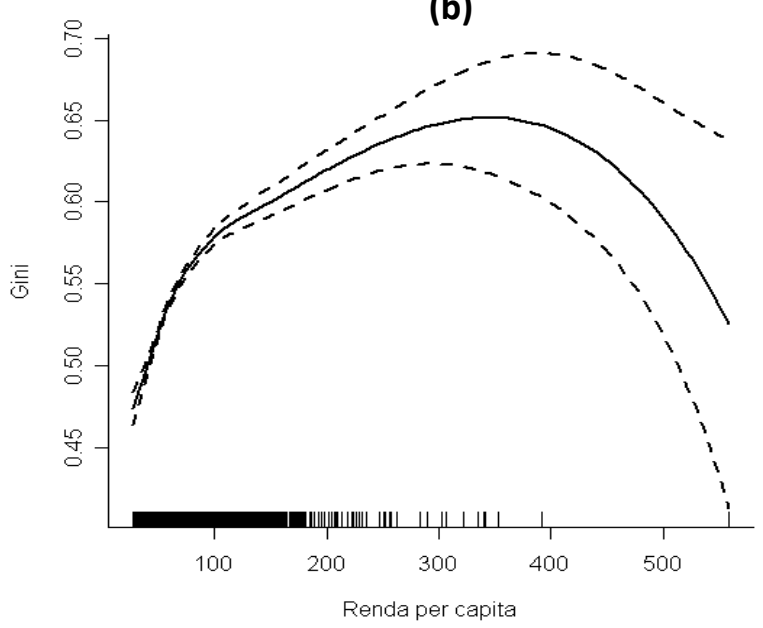

(d)

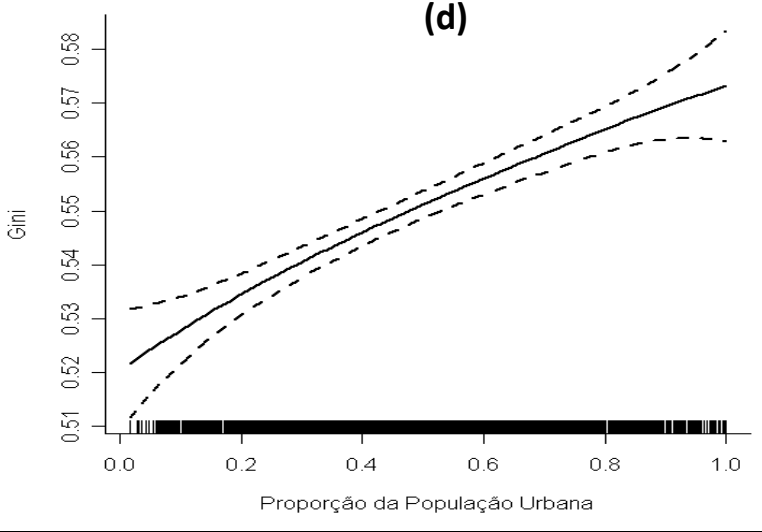

FONTE: Elaborados pelos autores.

O Gráfico 2 apresenta o alisamento Spline para a relação entre desigualdade e renda per capita e desigualdade para os quintis. Observa-se que os resultados obtidos nos modelos paramétricos e apresentados nas tabelas não são diferentes dos resultados apresentados nos gráficos. Ou seja, é uma forma alternativa, com modelos não paramétricos de validar a curva e Kuznets usando os critérios de Ahluwalia (1976). Como foi observado anteriormente, os 
modelos paramétricos tem uma vantagem relacionada ao fato de ser desnecessário impor uma estrutura, já que os dados falam por si.
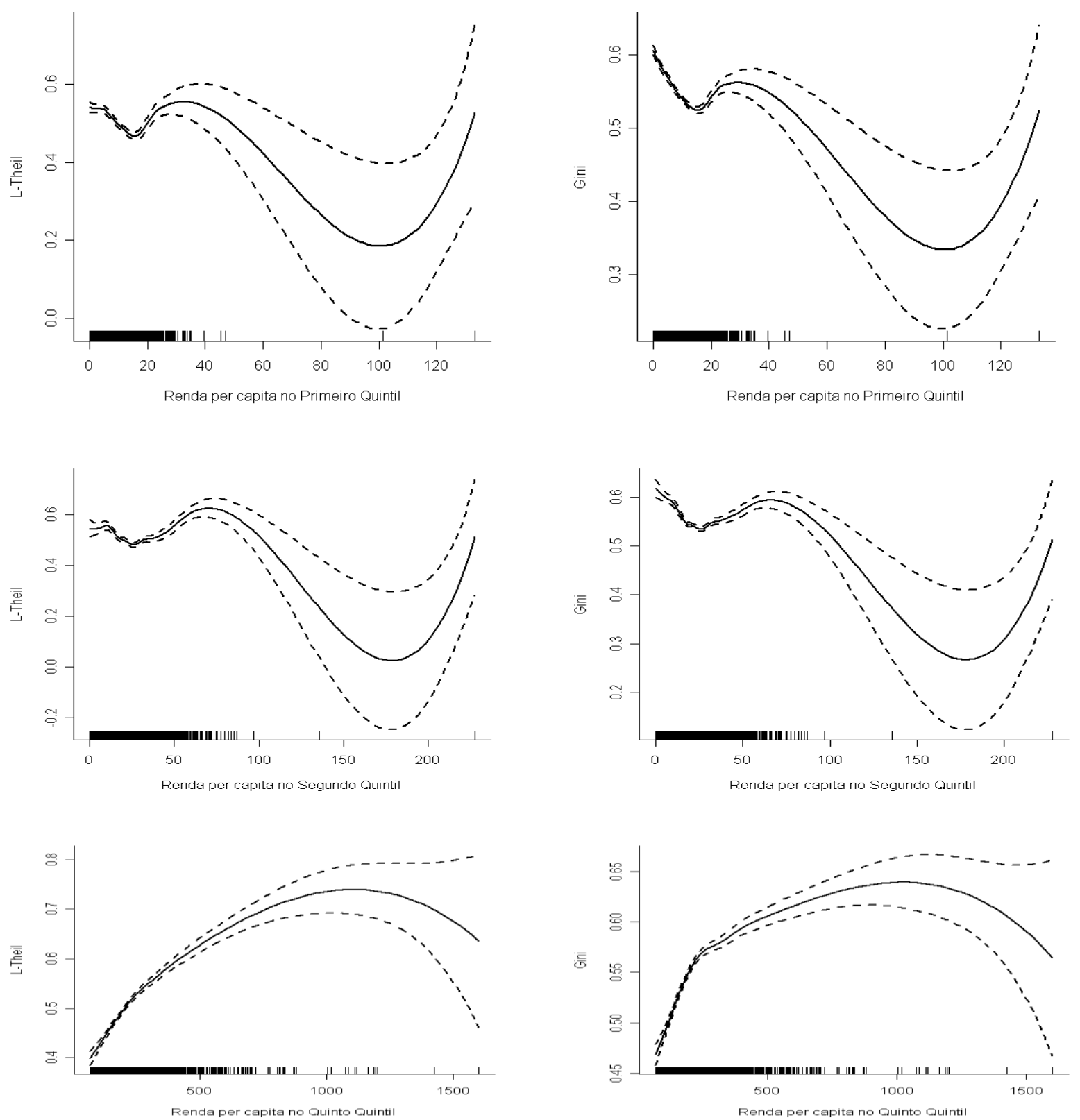

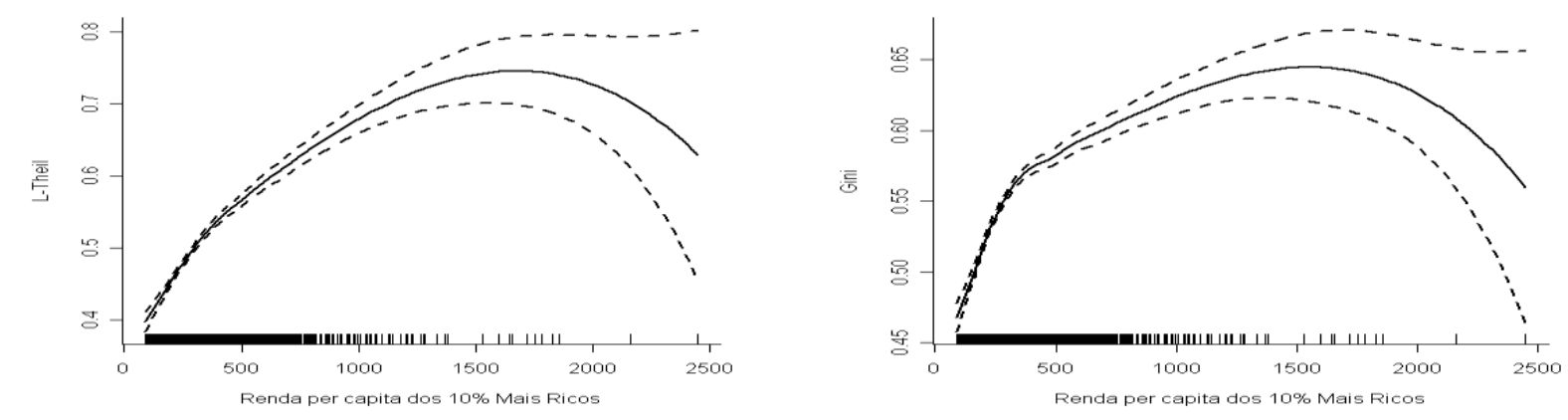

FONTE: Elaborados pelos autores.

\section{CONCLUSÕES}

As controvérsias em torno da validade da hipótese do U invertido foram e têm sido geradas por uma série de estudos nos quais dadas à natureza das informações, a especificação alternativa para a forma funcional e o método econométrico utilizado permitem validar ou não as conclusões de Kuznets. Independente dos resultados, esses estudos cumprem o papel de atender a necessidade de um conhecimento mais sólido e uma perspectiva mais convincente para a economia. Pode-se afirmar que, diferentemente de Kuznets (1955), esse trabalho foi resultado de $5 \%$ de especulação e 95\% de informação empírica para os municípios da região Nordeste do Brasil, cujo objetivo foi o de fornecer informações sobre a relação entre desigualdade e renda per capita.

Os resultados encontrados com o uso dos modelos semiparamétricos reforçam as evidências encontradas na literatura que empregaram modelos paramétricos. Como forma de tornar mais robustos esses resultados, também se buscou estimar o modelo para os quintis de uma maneira similar ao realizado por Ahluwalia (1976), ou seja, estimar os modelos para os quintis de menor renda para os de maior renda. As evidências obtidas no trabalho original de Ahluwalia (1976) são mantidas pelos modelos semiparamétricos.

\section{REFERÊNCIAS}

AGHION, P. e BOLTON, P. Distribution and Growth in Models of imperfect capital markets. European Economic Review. V. 36, p. 603-611, 1992.

AHLUWALIA, M. S. Income distribution and development: some stylized facts. American Economic Review. V. 66, p. 128-153, 1976a.

AHLUWALIA, M. S. Inequality, poverty and development. Journal of Development Sudies. V. 3, p. 307-342, 1976 b.

ALESINA, A. e RODRIK, D. Distributive politics and economic growth. Quarterly Journal of Economics. N. 109, p. 465-490, 1994. 
ANAND, S. e KANBUR, S. M. R. The Kuznets process and the inequality-development relationship. Journal of Development Economics. V. 40, p. 25-52, 1993a.

ARNAND, S. e KANBUR, S. M. R. Inequality and development: a critique. Journal of Development Economics. V. 41, p.19-43, 1993 b.

FUNDAÇÃO JOÃO PINHEIRO, IBGE E IPEA. Atlas de Desenvolvimento Humano. Belo Horizonte, 1996.

ENCONTRO NACIONAL DE ECONOMIA, 30, 2002, Nova Friburgo. Crescimento e Desigualdade no Rio Grande do Sul: uma revisão da Curva de Kuznets para os municípios gaúchos (1970-1991). Nova Friburgo, 2002.

BANERJEE, A. e NEWMAN, A. F. Risk-bearing and the theory of income distribution. Review of Economics Studies. V. 58, p. 211-235, 1991.

BARRETO, F. A. F. D., JORGE NETO, P. M. e TEBALDI, E. Desigualdade de renda e crescimento econômico no nordeste brasileiro. Revista Econômica do Nordeste, v. 32, n. Especial, P. 842-859, 2001.

BARRO, R. J. Inequality, growth and investment. NBER. Disponível em: <http://www.nber.org/papers/w7038>, 1999.

ENCONTRO DE ECONOMIA GAÚCHA, 1, 2002, Porto Alegre. A desigualdade econômica do Rio Grande do Sul: primeiras investigações sobre a curva de Kuznets. Porto Alegre, Maio, 2002.

BOURGUIGON, F. Growth and inequality in the dual model of development: the role of demand factors. Review of Economics Studies. V. 57, p. 215-228, 1990.

DEINIGNER, K. e SQUIRE, L. New ways of looking at the ols issues: inequality and growth. Journal of Development Economics, v. 57, p. 259-287, 1998.

DOEPKE, M. Fertility, income distribution and growth. (mimeo) University of Chicago. Disponível em: < http://chicago.edu/papers>, 1999.

DUARTE, R. Dinâmica e transformação da economia nordestina na década de 70 e nos anos 80. Revista Econômica do Nordeste, v. 33 n. Especial, p. 402-421, julho, 2002.

FERREIRA, F. H. G. Education for the masses?: The interaction between wealth, educational and political inequalities. Economics of Transistion. V. 9, no. 2, 2001.

FIELDS, G. S. Poverty, inequality and development. New York. Cambridge Press, 1980.

FIELDS, G. S. Distribution and Development a new look at the developing world. MIT Press, 2001.

FIELDS, G. S. e JAKUBSON, G. H. (1994), New evidence on the Kuznets curve. (Mimeo). Cornell University.

FRENCH, J. KAMMANN, E. e WAND, M. Comment on Ke and Wang. Journal of the American Statistical Association, v. 96, p. 1285-1288, 2001.

GREENE, W. Econometric analysis. Prentice Hall, New York, 2000.

HAUSMAN, J. A. Specification test in econometrics. Econometrica, v.46, n.6, p.1251-271, 1978.

JOHNSTON, J. E DINARDO, R. Econometric methods, 4th. Ed. New York: McGrawHill, 1994. 
KANBUR, R. Income distribution and development. (mimeo). Cornell University, 1999.

KUZNETS, S. Economic growth and income inequality. American Economic Review. V. 45, p.1-28, 1955.

LLEDÓ, V. D. Distribuição de renda, crescimento endógeno e política fiscal: uma análise cross-section para os estados brasileiros. Texto para Discussão n. 441. IPEA, Rio de Janeiro, 1996.

MADDALA, G.S. Introduction to econometrics. Second edition. Prentice Hall, New York. 1992.

PERSON, T. e TABELLINI, G. Is inequality harmful to growth? American economic Review. V. 84, p. 600-621, 1994.

PÕRTO JÚNIOR, S. S. E RIBEIRO, E. P. Dinâmica espacial da renda per capita e crescimento entre os municípios da região nordeste do Brasil - uma análise markoviana. Revista Econômica do Nordeste, v. 34, no. 3, p. 405-420, julho-setembro, 2003.

RAY, D. Development economics. Princeton University Press. Princeton. 1999.

ROBINSON, S. A note on the U hiphotesis relating inequality and economic development. American Economic Review. V. 66, p. 437-440, 1976.

ENCUENTRO DE ECONOMÍA APLICADA, 5, 2002, Oviedo. Desigualdad y crescimiento econômico: um estúdio analítico y empírico del proceso de Kuznets. Oviedo, 2002.

ENCONTRO NACIONAL DE ECONOMIA, 30, 2002, Nova Friburgo. Convergência, desigualdade e concentração de renda nas microregiões do nordeste brasileiro: 19701998. Nova Friburgo, 2002.

RUPPERT, D. e CARROLL, R. Spatially-adaptive penalties for spline fitting. Australian and New Zealand Journal of Statistics, v. 42, p. 205-224, 2000.

RUPPERT, D. WAND, M. e CARROLL, R. Semiparametric Regression. Cambridge: Cambridge series statistical and probabilistic mathematics, 416 pg, 2003.

SAINT-PAUL, G. e VERDIER, T. Education, democracy and Growth. Journal of Development Economics. V. 42, p. 399-407, 1993.

SILVEIRA NETO, R. M. e CAMPÊLO, A. K. Radiografando as disparidades regionais de renda no Brasil: evidências a partir de regressões quantílicas. Revista Econômica do Nordeste, v. 34 no. 3, p. 359-378, julho-setembro, 2003.

THORNTON, J. The Kuznets inverted-U hypothesis: panel data evidence from 96 countries. Applied Economics Letters, v. 8, p. 15-16, 2001.

ENCONTRO NACIONAL DE ECONOMIA, 24, 1996, Águas de Lindóia. Crescimento econômico e convergência da renda nos estados do Nordeste brasileiro. Águas de Lindóia, 1996.

WILLIANSON, J. G. Growth, distribution and demography: some lessons from history. Exploration in Economics History. No. 35, p. 241-271, 1998.

WOOLDRIDGE, J. Econometric analysis of cross section and panel data. MIT Press. Massachusetts, 2001 


\section{ANEXOS}

TABELA 1: SUMÁRIO DOS ESTUDOS SOBRE A CURVA DE KUZNETS

\begin{tabular}{c|r|}
\hline Referência & Âmbito do estudo \\
\hline Ahluwalia (1976) & $\begin{array}{r}\text { Amostra de } 60 \text { países sendo } \\
\text { que eram } 40 \text { considerados } \\
\text { como subdesenvolvidos, } 6 \\
\text { eram socialistas e } 14 \text { eram } \\
\text { desenvolvidos }\end{array}$ \\
Braulke (1983) & $\begin{array}{r}\text { Amostra de } 33 \text { países com } \\
\text { renda homogênea }\end{array}$
\end{tabular}

\author{
Método \\ Cross-section para \\ desigualdade em função da \\ renda per capita e uma \\ dummy para países \\ socialistas
}

Modelo não linear para o

Índice de Gini na função de razões setoriais de renda e população

$\operatorname{Ram}(1989)$

Anand e Kambur (1993b)

Fields e Jakubson (1994)

Hsing e Smyth (1994)

Dawson (1997)

Deininger e Squire (1998)

Ogwang (2000)

Sylvester (2000)
Amostra de 60 países (dados de Ahluwalia (1976)

Amostra com 115 países para período $1960-80$

Cross-section para um modelo quadrático do Índice de Theil em função do pib per capita

Croos-section para as funcionais derivadas para 6 índices de desigualdade em função do pib per capita

Amostra de 20 paísess

Cross-section Pooled e dados de painel para o modelo quadrático do índice de

Gini como função da renda per capita

Séries de tempo para economia americana no período 1948-87

Amostra dos 20 países menos desenvolvidos de

RAM (1989)

Amostra de 108 países para o período 1960-90

Estimação de um SUR para índice de Gini considerando

separadamente as raças brancas e negras

Cross-section com modelos quadráticos e semi-log para o coeficiente do Gini com respeito a renda per capita

Cross-section para o crescimento em função da renda, inverso da desigualdade e educação. Dados em painel do nível de desigualdade em função da renda média e do sistema político do país

Cross-section da desigualdade entre países para pib per capita e vários índices de desenvolvimento humano

Cross-section do crescimento em função dos gastos em (dados d

(1994)) educação e desigualdade de renda (índice de Gini)
Quando é considerada a convergência entre as rendas setoriais o $U$ invertido apresenta uma redução em sua fase inicial

Os resultados apoiam a hipótese do U invertido

Os resultados não apoiam a hipótese do U invertido

Os resultados do cross-section pooled apóiam a existência do U invertido, porém as estimações com efeitos fixos contradiz essa hipótese

Os resultados apoiam a hipótese do U invertido para as duas raças e o turning point coincide

Os resultados apoiam a hipótese do U invertido

A desigualdade reduz o crescimento econômico nos países pobres, mas não nos ricos. Os dados de séries de tempo apoiam a hipótese do $\mathrm{U}$ invertido

A relação da desigualdade e pib per capita com é maior do que com relação aos índices de desenvolvimento humano

Os gastos em educação afetam o crescimento no longo prazo e a desigualdade condiciona o crescimento a curto prazo 


\begin{tabular}{|c|c|c|c|}
\hline Referência & Âmbito do estudo & Método & Conclusões \\
\hline $\begin{array}{l}\text { Bagoli, Gabe e } \\
\text { Ribeiro (2004) }\end{array}$ & $\begin{array}{r}\text { Amostra dos municípios do } \\
\text { estado do Rio Grande do } \\
\text { Sul (1970, } 1980 \text { e } 1991)\end{array}$ & $\begin{array}{r}\text { Cross-section e dados em } \\
\text { painel do modelo } \\
\text { quadrático da desigualdade } \\
\text { em função da renda per } \\
\text { capita }\end{array}$ & $\begin{array}{r}\text { Resultados apoiam a hipótese } \\
\text { do U invertido. }\end{array}$ \\
\hline Jacinto e Tejada & $\begin{array}{r}\text { Amostra dos municípios da } \\
\text { região Nordeste do Brasil } \\
(1970,1980 \text { e 1991) }\end{array}$ & $\begin{array}{r}\text { Cross-section, pooled e dados } \\
\text { em painel para modelo } \\
\text { quadráticos e especifiçaões } \\
\text { altelternativas para o índice } \\
\text { d desigualdade }\end{array}$ & $\begin{array}{l}\text { Os resultados apoiam a } \\
\text { hipótese de U invertido. }\end{array}$ \\
\hline Salvato et al (2006) & $\begin{array}{r}\text { Amostra dos municípios do } \\
\text { estado de Minas Gerais } \\
(1991 \text { e } 2000)\end{array}$ & $\begin{array}{r}\text { Cross-section e dados em } \\
\text { painel do modelo } \\
\text { quadrático da desigualdade } \\
\text { em função da renda per } \\
\text { capita }\end{array}$ & $\begin{array}{l}\text { Os resultados apoiam a } \\
\text { hipótese de U invertido. }\end{array}$ \\
\hline $\begin{array}{l}\text { Barros e Reis } \\
\text { Gomes (2007) }\end{array}$ & $\begin{array}{r}\text { Amostra dos municípios do } \\
\text { Brasil (1991 e 2000) }\end{array}$ & Pooled e dados em painel & $\begin{array}{l}\text { O resultados apoiam a } \\
\text { hipótese de } U \text { invertido }\end{array}$ \\
\hline
\end{tabular}

